\title{
On the Apparent Lack of Be X-ray Binaries with Black Holes
}

\section{Krzysztof Belczyński}

Los Alamos National Lab, P.O. Box 1663, MS 466, Los Alamos, NM 87545

E-mail: kbelczynenmsu.edu

\section{Janusz Ziółkowski*}

Copernicus Astronomical Center, Warsaw, Poland

E-mail: jz@camk.edu.pl

In the Galaxy there are $64 \mathrm{Be} \mathrm{X}$-ray binaries known to-date. Out of those, 42 host a neutron star, and for the reminder the nature of a companion is not known. None, so far, is known to host a black hole. There seems to be no apparent mechanism that would prevent formation or detection of Be stars with black holes. This disparity is referred to as a missing Be - black hole $\mathrm{X}$-ray binary problem. We point out that current evolutionary scenarios that lead to the formation of Be X-ray binaries predict that the ratio of these binaries with neutron stars to the ones with black holes is rather high $F_{\mathrm{NStoBH}} \sim 30-50$. The ratio is a natural outcome of $(i)$ the stellar initial mass function that provides more neutron stars than black holes and (ii) common envelope evolution (i.e. a major mechanism involved in the formation of interacting binaries) that naturally selects progenitors of Be X-ray binaries with neutron stars (comparable mass binaries have more likely survival probabilities) against ones with black holes (much more likely common envelope mergers). A comparison of this ratio (i.e. $F_{\mathrm{NStoBH}} \sim 30-50$ ) with the number of confirmed Be - neutron star X-ray binaries (42) indicates that the expected number of Be - black hole X-ray binaries is of the order of only $\sim 0-2$. This is entirely consistent with the observed Galactic sample.

The Extreme Sky: Sampling the Universe above $10 \mathrm{keV}$

October 13-17 2009

Otranto, Italy

\footnotetext{
* Speaker.
} 


\section{Introduction}

High mass X-ray binaries host a compact object (a neutron star or a black hole) and a massive star. The major subclass of high mass X-ray binaries consists of a Be star and a compact object and they are referred to as Be X-ray binaries. The Be stars are massive, generally main sequence, stars of spectral types A0-O8 with Balmer emission lines (Negueruela 1998). The Be XRBs are found with rather wide (orbital periods in the range of $\sim 10-300$ days) and frequently eccentric orbit and a compact object accretes from the wind of a Be star (even massive Be stars are within their Roche lobes for these wide orbits). At present, 64 Be XRBs are known in the Galaxy, and in 42 the compact object was confirmed to be a neutron star (NS) by the presence of the X-ray pulsations. In the remaining cases, whenever we have information concerning the nature of the compact component (such as an X-ray spectrum), it also indicates a NS. Although one cannot exclude that a few of these systems contain white dwarfs or black holes, it is fair to state that majority of them contain NSs as compact components. We know, at present, 55 black hole candidate systems in the Galaxy (among them 19 confirmed BH systems; e.g., Remillard \& McClintock 2006; Ziółkowski 2008). However, not a single black hole binary containing a Be type component has been found so far. This disparity, 42 Be XRBs with NSs versus not a single one with a BH, seems indeed striking.

The X-ray emission from Be XRBs (with a few exceptions) is of a distinctly transient nature with rather short active phases separated by much longer quiescent intervals (a flaring behavior). There are two types of flares, which are classified as Type I outbursts (smaller and regularly repeating) and Type II outbursts (larger and irregular; Negueruela \& Okazaki 2001, Negueruela et al. 2001). Type I bursts are observed in systems with highly eccentric orbits. They occur close to periastron passages of a NS. They are repeating at intervals $\sim P_{\text {orb }}$. Type II bursts may occur at any orbital phase. They are correlated with the disruption of the excretion disc around Be star (as observed in $\mathrm{H} \alpha$ line). They repeat on time scale of the dynamical evolution of the excretion disc ( $\sim$ few to few tens of years). This recurrence time scale is generally much longer than the orbital period (Negueruela et al. 2001).

Be XRBs systems are known to contain two discs: excretion disc around Be star and accretion disc around neutron star. Both discs are temporary: excretion disc disperses and refills on time scales $\sim$ few to few tens of years (dynamical evolution of the disc, formerly known as the "activity of a Be star" (Negueruela et al. 2001)), while the accretion disc disperses and refills on time scales $\sim$ weeks to months (which is related to the orbital motion on an eccentric orbit and, on some occasions, also to the major instabilities of the other disc). The accretion disc might be absent over a longer period of time ( $\sim$ years), if the other disc is very weak or absent. The X-ray emission of $\mathrm{Be} \mathrm{XRBs}$ binaries is controlled by the centrifugal gate mechanism, which, in turn, is operated both by the periastron passages (Type I bursts) and by the dynamical evolution of the excretion disc (both types of bursts). This mechanism explains the transient nature of the X-ray emission ( see Ziółkowski 2002 and references therein).

In this work we study the origins of the apparent disparity of number of known Be XRBs with NSs (42) as compared to no known Be XRBs with BHs in Galaxy. This disparity has been noted in the literature for some time. First stellar population synthesis calculations intented to estimate the number of black hole Be X-ray binaries were carried out by Raguzova \& Lipunov (1999). They assumed that the observed value of $F_{\mathrm{NStoBH}}$ is $\sim 25$. This was based on $\sim 25$ galactic NS Be X- 
ray binaries known at that time and the assumption that GRS $1915+105$ is a BH Be X-ray binary (which, as we know now, is not true). They demonstrated that by adjusting parameters such as the value of the lower mass limit for the formation of black hole $M_{\mathrm{cr}}$ and value of the kick velocity during the $\mathrm{SN}$ explosion, one can reproduce the observed value of $\sim 25$. However, their value of $M_{\text {cr }}\left(55\right.$ to $75 \mathrm{M}_{\odot}$ ) seems much to high, according to present knowledge. Also their assumption that each main sequence star above $10 \mathrm{M}_{\odot}$ becomes a Be star after a mass accretion episode does not have to be necessarily true.

\section{Stellar Population Synthesis (SPS) Calculations}

\subsection{SPS Code}

We evolve a Galactic population of massive binaries using StarTrack stellar population synthesis code (Belczyński, Kalogera \& Bulik 2002 and Belczyński et al. 2008). We adopt solar metallicity $(Z=0.02)$, and a steep initial mass function (IMF) for massive stars with a power-law exponent of -2.7 (Kroupa \& Weidner 2003). Roche lobe overflow is treated in a non-conservative way (with 50\% mass loss from a given binary; e.g. Meurs \& van den Heuvel 1989) while the CE phase is treated via energy balance with fully efficient transfer of orbital energy into dispersal of an envelope (e.g. $\alpha \times \lambda=1.0$ ). The results are calibrated in such a way that the Galactic star formation rate is at the level of $3.5 \mathrm{M}_{\odot} / \mathrm{yr}$ and is constant through the last $10 \mathrm{Gyr}$ (e.g. O'Shaughnessy et al. 2008). At the present Galactic disk age $(t=10 \mathrm{Gyr})$ we perform a time slice and extract Be X-ray binaries using classification criteria defined in the following section.

\subsection{Definition of a Be XRB for the purpose of SPS calculations}

During our SPS calculations, we consider any system a Be X-ray binary if: (i) it hosts either a NS or a BH accretor; (ii) donor is a main sequence star (burning $\mathrm{H}$ in its core); (iii) donor mass is higher than $3 \mathrm{M}_{\odot}\left(\mathrm{O} / \mathrm{B}\right.$ star); (iv) orbital period is in the range $10 \leq P_{\text {orb }} \leq 300$ day; and ( $v$ ) only a fraction $F_{\mathrm{Be}}=0.25$ of the above systems are designated as hosting a Be star and not a regular $\mathrm{O} / \mathrm{B}$ star.

The last condition is based on the observations indicating that the fraction of Be stars among all B stars is $1 / 5$ to $1 / 3$ (e.g., Ziółkowski 2002; McSwain \& Gies 2005).

Our set of conditions means that we assume that whenever donor is a Be star then the accretion is always efficient, independently of the size of the binary orbit (as is, in fact, observed in Be/NS XRBs).

\subsection{SPS Models}

We carried out the calculations for three models of SPS. In model A, it was assumed that the binary system will survive the situation when the donor star will overflow its Roche lobe while crossing the Hertzsprung gap. With the present state of knowledge, it seems doubtful, as this would rather lead to a merger of both components (Taam \& Sandquist 2000, Ivanova \& Taam 2004). However, since model A used to be a standard in the past, we still carried out the calculations for this case. More realistic seem to be models B and C, which assume that overflowing by a donor its Roche lobe while crossing the Hertzsprung gap, leads to a merger and removal of the 
binary from the statistics. The difference between the models $\mathrm{B}$ and $\mathrm{C}$ concerns the natal kicks compact objects receive at birth. Model B assumes for NSs the kicks drawn from the radio pulsar birth velocity distribution derived by Hobbs et al. (2005; a Maxwellian with $\sigma=265 \mathrm{~km} / \mathrm{s}$ ). However, there are some indications that natal kicks neutron stars receive are smaller for stars in binaries as compared to single stars (e.g. Podsiadlowski et al. 2004). Therefore, model C assumes a Maxwellian distribution with $\sigma=133 \mathrm{~km} / \mathrm{s}$.

\section{Results}

For model $\mathrm{A}$ the expected ratio of Be-X binaries with $\mathrm{NSs}$ to the ones with $\mathrm{BHs}, F_{\mathrm{NStoBH}}$ was found to be $\sim 7$. For, more physically realistic, models $\mathrm{B}$ and $\mathrm{C}$, this ratio was found to be, respectively, 27 and 54. This relatively high ratio (for models B and C) is a natural outcome of $(i)$ the stellar initial mass function that provides more neutron stars than black holes and (ii) common envelope evolution (i.e. a major mechanism involved in the formation of interacting binaries) that naturally selects progenitors of Be X-ray binaries with neutron stars (comparable mass binaries have more likely survival probabilities) against ones with black holes (much more likely common envelope mergers).

The expected distributions of orbital periods and eccentricities for Be/NS and Be/BH binaries for model $\mathrm{C}$ are shown in Fig. 1. For comparison, the observed orbital period distribution for 27 galactic Be/NS binaries is shown in Fig. 2.

More detailed description of the results is given in Belczyński \& Ziółkowski (2009)

\section{Conclusions}

Our main results may be described as follows:

- We predict that both population of Be X-ray binaries should exist in the Galaxy: those with NSs as well as these with BHs.

- The predicted number of Be X-ray binaries with NSs is much higher (factors of $F_{\mathrm{NStoBH}} \sim$ $10-50)$ than those with BHs.

- If we use the preferred evolutionary models $\left(F_{\mathrm{NStoBH}} \sim 30-50\right.$; models B and C) we predict that in the observed sample of Be X-ray binaries of 42 systems with NSs, one should expect only $\sim 0-2$ systems with BHs. It is quite possible that none are yet observed (small statistics).

- Due to a very low number of expected binaries with BHs, it is very likely that there is no problem with missing Be X-ray binaries with BHs in Galaxy.

\section{Acknowledgments}

JZ acknowledges the support from the Polish Ministry of Science and Higher Education (MSHE) project 362/1/N-INTEGRAL (2009-2012) and KB acknowledges the support from MSHE grant N N203 302835 (2008-2011). 

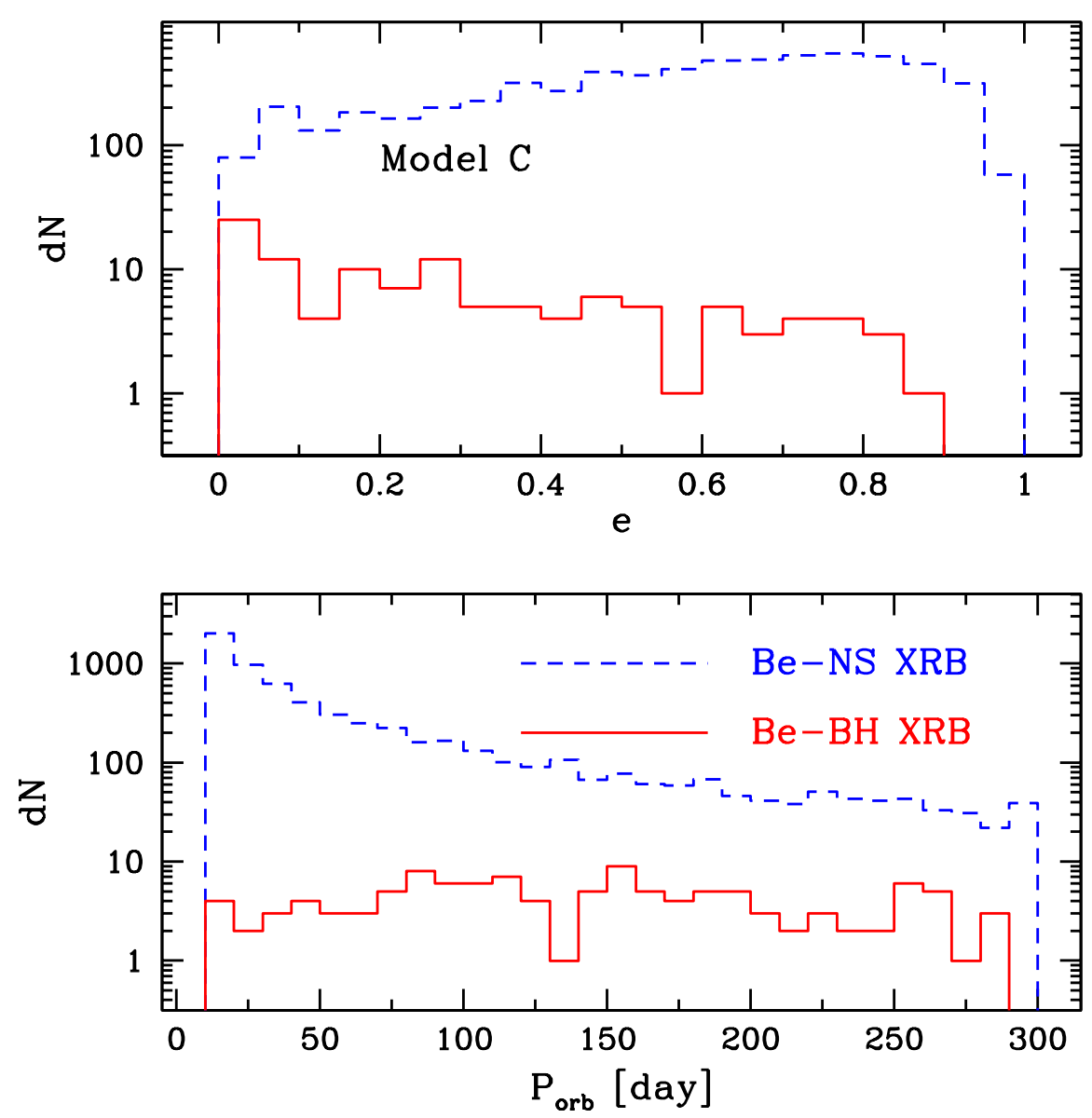

Figure 1: Expected orbital period and eccentricity distributions for Be/NS (blue line) and Be/BH (red line) binaries for model $\mathrm{C}$ of stellar population synthesis (see the text).

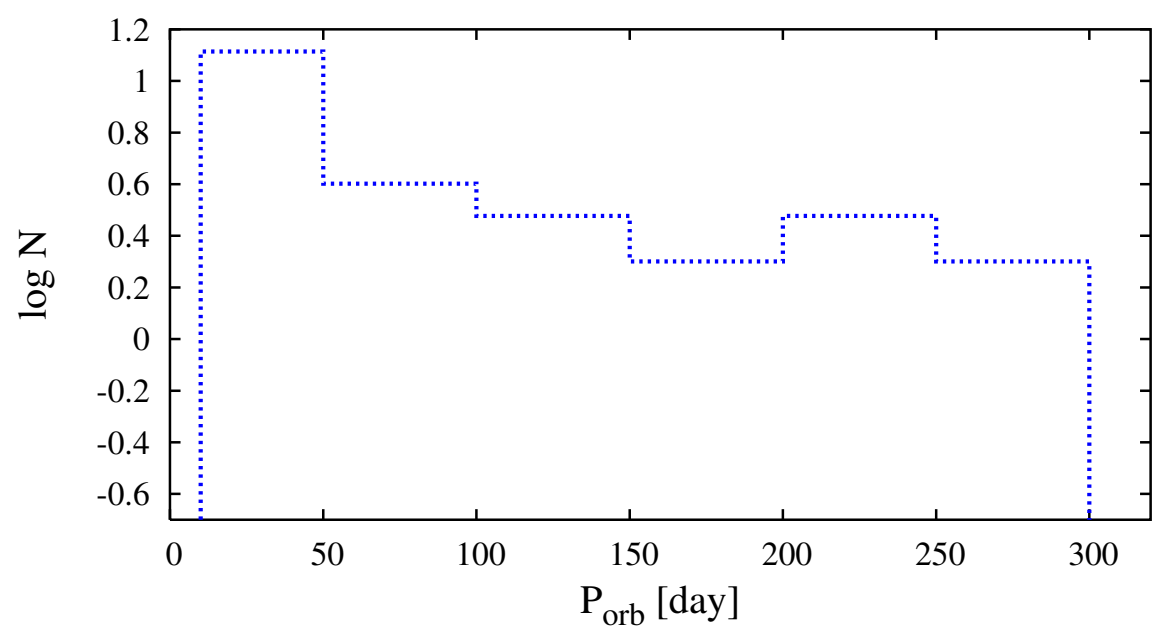

Figure 2: Observed orbital period distribution for 27 galactic Be/NS binaries. 


\section{References}

[1] P. Artymowicz \& S.H. Lubow, Dynamics of binary-disk interaction. 1: Resonances and disk gap sizes, ApJ 421 (1994) 651

[2] K. Belczyński, V. Kalogera \& T. Bulik, Binary Population Synthesis: Methods, Normalization, and Surprises, ApJ 572 (2002) 407

[3] K. Belczyński, V. Kalogera, F.A. Rasio, R.E. Taam, A. Zezas, T. Bulik, T.J. Maccarone \& N. Ivanova, Compact Object Modeling with the StarTrack Population Synthesis Code, ApJ Suppl 174 (2008) 223

[4] K. Belczyński \& J. Ziółkowski, On the Apparent Lack of Be X-ray Binaries with Black Holes, ApJ (2009) in press, [astro-ph/0907.4990]

[5] G. Hobbs, D.R. Lorimer, A.G. Lyne \& M. Kramer, A statistical study of 233 pulsar proper motions, MNRAS 360 (2005) 974

[6] W. Hummel \& R.W. Hanuschik, Line formation in Be star envelopes. II. Disk oscillations, A\&A 320 (1997) 852

[7] N. Ivanova \& R.E. Taam, Thermal Timescale Mass Transfer and the Evolution of White Dwarf Binaries, ApJ 601 (2004) 1058

[8] P. Kroupa \& C. Weidner, Galactic-Field Initial Mass Functions of Massive Stars, ApJ 598 (2003) 1076

[9] M.V. McSwain \& D.R. Gies, The Evolutionary Status of Be Stars: Results from a Photometric Study of Southern Open Clusters, ApJ Suppl 161 (2005) 118

[10] E.J.A. Meurs \& E.P.J. van den Heuvel, The number of evolved early-type close binaries in the Galaxy, $A \& A 226$ (1989) 88

[11] I. Negueruela, On the nature of Be/X-ray binaries, $A \& A 338$ (1998) 505

[12] I. Negueruela \& A.T. Okazaki, The Be/X-ray transient 4U 0115+63/V635 Cas: I. A consistent model, $A \& A 369$ (2001) 108

[13] I. Negueruela, A.T. Okazaki, J. Fabregat, M.J. Coe, U. Munari \& T. Tomov, The Be/X-ray transient 4U 0115+63/V635 Cas: II. Outburst mechanisms, A\&A 369 (2001) 117

[14] R. O’Shaughnessy, C. Kim, V. Kalogera \& K. Belczynski, Constraining Population Synthesis Models via Empirical Binary Compact Object Merger and Supernova Rates, ApJ 672 (2008) 479

[15] Ph. Podsiadlowski, N. Langer, A.J.T. Poelarends, S. Rappaport, A. Heger \& E.D. Pfahl, The Effects of Binary Evolution on the Dynamics of Core Collapse and Neutron Star Kicks, ApJ 612 (2004) 1044

[16] Ph. Podsiadlowski, S. Rappaport \& Z. Han, On the formation and evolution of black hole binaries, MNRAS 341 (2003) 385

[17] N.V. Raguzova \& V.M. Lipunov, The evolutionary evidence for Be/black hole binaries, A\&A 349 (1999) 505

[18] R.A. Remillard \& J.E. McClintock, X-ray Properties of Black-Hole Binaries, ARA\&A 44 (2006) 49

[19] R.E. Taam \& E.L. Sandquist, Common Envelope Evolution of Massive Binary Stars, ARA\&A 38 (2000) 113

[20] F. Zhang, X.-D. Li \& Z.-R. Wang, Where Are the Be/Black Hole Binaries?, ApJ 603 (2004) 663

[21] J. Ziółkowski, Be/X-ray Binaries, Mem. Soc. Astron. Ital. 73 (2002) 1038

[22] J. Ziółkowski, Masses of Black Holes in the Universe, Chin. J. Astron. Astrophys. 8 Suppl. (2008) 273 